\title{
ARTICLE
}

Clinical nutrition

\section{Mucosal lactoferrin response to genital tract infections is associated with iron and nutritional biomarkers in young Burkinabé women}

\author{
S. A. Roberts ${ }^{1}$ - L. Brabin ${ }^{2}$ - S. Diallo - S. Gies ${ }^{4}$ A. Nelson ${ }^{5}$ - C. Stewart ${ }^{6}$ D. W. Swinkels ${ }^{7,8} \cdot$ A. J. Geurts-Moespot ${ }^{7}$. \\ A. Kazienga ${ }^{3} \cdot$ S. Ouedraogo ${ }^{3} \cdot$ U. D'Alessandro $^{9,10} \cdot$ H. Tinto $^{3} \cdot$ B. J. Brabin ${ }^{11,12}$
}

Received: 27 February 2019 / Revised: 29 April 2019 / Accepted: 19 May 2019 / Published online: 5 June 2019

(c) The Author(s) 2019. This article is published with open access

\begin{abstract}
Background/Objectives The iron-binding affinity of vaginal lactoferrin (Lf) reduces iron available to genital pathogens. We describe host reproductive, nutritional, infection and iron biomarker profiles affecting vaginal Lf concentration in young nulliparous and primigravid women in Burkina Faso.

Subjects/Methods Vaginal eluates from women who had participated in a randomized, controlled periconceptional iron supplementation trial were used to measure Lf using a competitive double-sandwich ELISA. For this analysis samples from both trial arms were combined and pregnant and non-pregnant cohorts compared. Following randomization Lf was measured after 18 months (end assessment) for women remaining non-pregnant, and at two antenatal visits for those becoming pregnant. Associations between log Lf levels and demographic, anthropometric, infection and iron biomarker variables were assessed using linear mixed models.

Results Lf samples were available for 712 non-pregnant women at end assessment and for 303 women seen at an antenatal visit. Lf concentrations of pregnant women were comparable to those of non-pregnant, sexually active women. Lf concentration increased with mid-upper-arm circumference, $(P=0.047)$, body mass index $(P=0.018)$, Trichomonas vaginalis $(P<0.001)$ infection, bacterial vaginosis $(P<0.001)$, serum $\mathrm{C}$-reactive protein $(P=0.048)$ and microbiota community state types III/IV. Adjusted Lf concentration was positively associated with serum hepcidin $(P=0.047)$, serum ferritin $(P=$ $0.018)$ and total body iron stores $(P=0.042)$. There was evidence that some women maintained persistently high or low $\mathrm{Lf}$ concentrations from before, and through, pregnancy.

Conclusion Lf concentrations increased with genital infection, higher BMI, MUAC, body iron stores and hepcidin, suggesting nutritional and iron status influence homeostatic mechanisms controlling vaginal Lf responses.
\end{abstract}

Supplementary information The online version of this article (https:// doi.org/10.1038/s41430-019-0444-7) contains supplementary material, which is available to authorized users.

B. J. Brabin

b.j.brabin@liverpool.ac.uk

1 Centre for Biostatistics, Division of Population Health, Health Services Research and Primary Care, Faculty of Biology, Medicine and Health, School of Medical Sciences, University of Manchester, Manchester Academic Health Science Centre, Manchester, UK

2 Division of Cancer Sciences, School of Medical Sciences, Faculty of Biology, Medicine and Health, University of Manchester, Manchester Academic Health Science Centre, Manchester, UK

3 Clinical Research Unit of Nanoro (URCN/IRSS), Nanoro, Burkina Faso

4 Department of Biomedical Sciences, Prince Leopold Institute of Tropical Medicine, Antwerp, Belgium and Medical Mission Institute, Würzburg, Germany
5 Faculty of Health and Life Sciences, University of Northumbria, Northumberland Building, Newcastle-upon-Tyne, UK

6 Molecular Virology and Microbiology, Baylor College of Medicine, Houston, TX, USA

7 Department of Laboratory Medicine (TLM 830), Radboud University Nijmegen Medical Center, Nijmegen, The Netherlands

8 Hepcidinanalysis.com., Nijmegen, The Netherlands

9 Medical Research Council Unit (MRC), Fajara, The Gambia

10 London School of Hygiene and Tropical Medicine, London, UK

11 Liverpool School of Tropical Medicine, and Institute of Infection and Global Health, University of Liverpool, Liverpool, UK

12 Global Child Health Group, Academic Medical Centre, University of Amsterdam, Amsterdam, The Netherlands 


\section{Introduction}

An essential role of human lactoferrin (Lf) is to prevent accumulation of free iron at mucosal sites. Lf demonstrates a bacteriostatic effect related to its iron-binding affinity [1], as well as antimicrobial activity that reduces bacterial virulence [2], inhibitory effects on bacterial adhesion and cell invasion, and ability to induce bacterial lysis $[3,4]$. Lf is expressed in the genital tract as part of the innate immune system, active against common genital tract infections such as Trichomonas $(T)$ vaginalis, bacterial vaginosis (BV)related species, and Candida spp. Iron is an essential nutrient for many genital tract bacteria [5], which have developed specialized mechanisms for obtaining iron from host tissues and extracellular fluid [6]. Iron is readily available from heme during menses, while transferrin and ferritin are transudated across the vagina lamina propria [7]. Lf concentrations rise with genital infection $[8,9]$ because neutrophils release $\mathrm{Lf}$ from their secondary compartment to sites of infection. Although Lf binds and reduces iron availability, Lf-bound iron may itself become an iron source for common bacteria such as Gardnerella vaginalis [10]. In systemic infection, proteins involved in iron homeostasis are regulated at the macrophage level during inflammation [11] and the interaction between ferroportin and hepcidin ensures that circulatory iron is related to host requirements. A local hepcidin response to genital tract inflammation has not been described. Independent of inflammation, Lf gene expression is also regulated by estrogen, which controls Lf produced constitutively in vaginal epithelial cells [3, 12]. Vaginal Lf concentrations vary over the menstrual cycle with estrogen surges [13], and plasma Lf was reported to be higher in pregnancy [14].

Given the lack of studies characterizing Lf profiles in women of reproductive age, we sought to describe factors affecting Lf concentration in a young, largely adolescent population participating in a periconceptional trial to investigate the safety of weekly iron supplementation in rural Burkina Faso. Results of this randomized trial have been reported $[15,16]$, including our finding of an effect modifier of nutritional status for BV infection. The aims of the present sub-study were (1) to measure and compare Lf concentrations from vaginal eluates in nulliparae and primigravidae; (2) to estimate the effects of participant characteristics and nutritional status, measured by body mass index (BMI) and mid-upper arm circumference (MUAC), on vaginal Lf concentration; (3) to estimate the effects of vaginal infections (BV, T. vaginalis, microbiota profiles), and (4) to compare systemic iron and inflammation biomarker concentrations, body iron stores, and hepcidin levels with vaginal $\mathrm{Lf}$ concentrations in order to determine if $\mathrm{Lf}$, a vaginal mucosal biomarker of infection, was associated with systemic iron status and homeostasis.

\section{Materials and methods}

The main trial recruited young, healthy, nulliparous, nonpregnant women aged 15-24 years from 30 rural villages [17]. Adolescents $(<20$ years) comprised $93 \%$ of the sample. Human immunodeficiency virus prevalence is low $(<2 \%)$ [18]. After enrolment individuals were randomized to receive either a capsule containing ferrous gluconate (60 mg elemental iron) and folic acid $(2.8 \mathrm{mg})$, or an identical capsule containing folic acid alone $(2.8 \mathrm{mg})$, as recommended by the World Health Organization, and investigators and recipients were blinded to allocation [19]. Field workers dispensed supplements at weekly home visits, and referred women with reported vaginal discharge or generalized symptoms for free treatment at their nearest Health Centre. Participants who became pregnant before the end assessment at 18 months entered the pregnant cohort, with the main trial outcomes determined at the first antenatal visit (ANC1). Thereafter, weekly supplements ceased and all pregnant women regardless of trial allocation received routine daily antenatal iron supplementation ( $60 \mathrm{mg}$ elemental iron, $400 \mu \mathrm{g}$ folic acid daily). In the primary trial analyses, weekly iron supplementation over an 18-month period did not significantly improve iron status, probably because the iron was not absorbed due to the effects of chronic malaria infection [15]; thus the two trial arms are pooled for the analyses presented here.

All subjects gave written informed consent in accordance with the Declaration of Helsinki. Individual and guardian written consents for minors were obtained from all nonpregnant women at recruitment with re-consent taken at entry to the pregnancy cohort. The study protocol and subsequent amendments were approved by the Liverpool School of Tropical Medicine, UK, Research Ethics Committee (LSTM/ REC), the Institutional Review Board of the Institute of Tropical Medicine, Antwerp, Belgium (IRB/ITM), the Antwerp University Hospital Ethics Committee (EC/UZA), the Institutional Ethics Committee of Centre Muraz (Comité d'Ethique Institutionnel du Centre Muraz, CEI/CM); and the National Ethics Committee (Comité Ethique pour la Recherche en Santé, CERS) in Burkina Faso.

At enrolment demographic data were recorded and height (nearest $\mathrm{mm}$ ), weight (nearest $100 \mathrm{~g}$ ), and MUAC (mm) measured in duplicate. $\mathrm{Lf}, \mathrm{BV}$ and $T$. vaginalis were assessed after 18 months at the end assessment of nonpregnant women and twice during pregnancy at an early/ late antenatal visit $(\mathrm{ANC} 1 / 2)$. Women were requested to provide self-taken vaginal samples using cotton-tipped swabs that were returned to sterile, sealed tubes (Probact Transport Swabs, Technical Services Consultants, Lancs, UK) and kept cool until brought to the laboratory within 2-4 h. Samples were not requested during menses. Reported discharge was recorded. One swab was used for a BV Gram 
stain and a second for measurement of vaginal $\mathrm{pH}(\mathrm{pH}$ indicator sticks ranged from 3.6-6.1), as previously described [16]. Gram stains were scored using Nugent criteria with 7-10 indicating $\mathrm{BV}, 4-6$ intermediate, and 0-3 normal flora. Duplicate swabs were retained for preparation of vaginal fluid eluates for $\mathrm{Lf}$ assays, microbiota and $T$. vaginalis Polymerase Chain Reaction assays [16]. For microbiota, bacterial profiling of the variable region 4 (V4) of the $16 \mathrm{~S}$ rRNA gene was performed by NU-OMICS (Northumbria University, UK) based on the Schloss wet-lab MiSeq procedure [20].

Each tube containing a swab for vaginal eluate was weighed before and after sampling and the weight difference between initial and final weights was recorded. On laboratory arrival $5 \mathrm{ml}$ phosphate buffered saline was added to the tube which was shaken for $5 \mathrm{~min}$ on high speed, before pipetting and freezing at $-20^{\circ} \mathrm{C}$. Lf concentration was measured by a two-site enzyme-linked immunosorbent assay (double antibody sandwich ELISA) specific to human Lf (Kamiya Biomedical Co, KT-489, Seattle, US). The intra-assay coefficient of variation was $9.7 \%$. Duplicate samples were processed and analyzed independently. The derived mean sample weight was $0.033 \mathrm{~g}$, although weights were highly variable and $18 \%$ were negative. Residuals from a linear regression of the difference in estimated weights between the repeat samples against the difference in Lf concentration suggested a sample weight measurement error (standard deviation) of $0.035 \mathrm{~g}$. Temporal variation in derived weights suggested a batch or seasonal effect and operator differences were not discerned. Mean Lf values were therefore normalised by mean weight for type of visit (allowing for pregnancy effects) derived from a linear model which included month as a covariate. The denominators used were ANC1: $0.038 \mathrm{~g}$; ANC2: $0.048 \mathrm{~g}$; end assessment: $0.038 \mathrm{~g}$. A dilution series was used and values above the ELISA assay range were assigned a nominal high value of $104 \mu \mathrm{g} / \mathrm{ml}$, and those with no Lf detected were assumed to have no fluid collected if the duplicate sample recorded a detectable level.

Hemoglobin was measured (Sysmex automated analyser) on fresh whole blood. Cut-off points for anaemia were $<12 \mathrm{~g} / \mathrm{dl}$ for non-pregnant and $<11 \mathrm{~g} / \mathrm{dl}$ for pregnant women. Methods for assessing iron biomarkers and Creactive protein (CRP) have been previously reported [21]. Briefly, plasma ferritin and transferrin receptor (sTfR) were measured in duplicate by ELISA (Spectro Ferritin S-22 and TFC 94 Transferrin Receptor, RAMCO Laboratories Inc, Texas, US). CRP was assessed by ELISA (EU59131, IBL International, GMBH, Hamburg, Germany) at the Nanoro Research Laboratories, with an upper limit of the normal range in non-pregnant individuals between 5 and $8 \mathrm{mg} / \mathrm{l}$. Iron deficiency was estimated using a ratio of $\mathrm{sTfR} \mu \mathrm{g} / \mathrm{ml}$ to $\log _{10}$ ferritin $>5.6$ [21]. Body iron stores (BIS) $(\mathrm{mg} / \mathrm{kg})$ were calculated using the equation derived by Cook et al. [22] body iron $(\mathrm{mg} / \mathrm{kg})=-\left[\log _{10}(1000 \times \mathrm{sTfR} /\right.$ ferritin $)-$ $2.8229] / 0.1207$, using adjusted ferritin based on an internal regression correction approach allowing for inflammation as described by Namaste et al. [23], based on all the trial data [21]. Serum hepcidin was measured by competitive ELISA assay as previously described [24].

\section{Statistical analysis}

No differences in vaginal infections [16] or Lf levels were detected by study arm in the formal intention-to-treat analysis (Supplementary file Table 1), and trial arms were pooled in all analyses presented here. Two primary analysis datasets were constructed: (1) pregnant women with $\mathrm{Lf}$ assessed at either ANC1 or ANC2 (303/315 with an ANC visit); and (2) women who remained non-pregnant at end assessment (712/819 of this cohort) (Fig. 1). Women with uncertain pregnancy status at end assessment were excluded from this data set. A subset of 53/73 women first identified as pregnant at, or within 6 months, of end assessment were screened again for Lf at ANC1 and ANC2 and are considered in a separate longitudinal analysis.

The contribution of each source of variation in Lf concentration was assessed using a linear random effects model with terms for participant and visit (within participant), the residual variance being that between the duplicate samples taken at the same time from the same woman. Means of the $\log (\mathrm{Lf})$ values derived from repeat samples collected at the same visit were used for further analysis. The associations between $\log (\mathrm{Lf})$ levels and demographic, anthropometric, infection, and iron biomarker variables were assessed using a linear mixed model, pooling all visits with a random effect term to allow for correlations between levels in the same individuals. A combined (non-pregnant and pregnant) regression slope estimate was used. As nutritional status was a priori identified as a potential confounder, a second model was fitted which included MUAC, a surrogate for nutritional

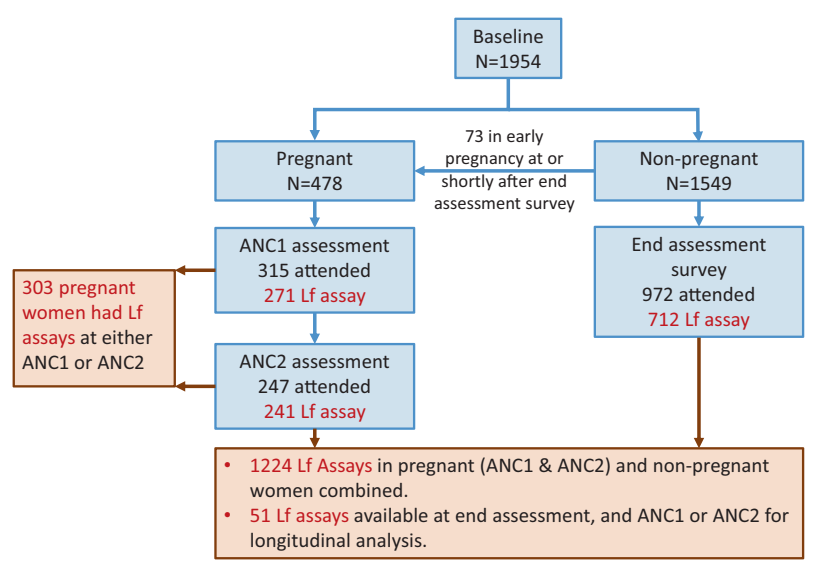

Fig. 1 Flow diagram indicating non-pregnant and pregnant study numbers and sample sizes for Lf assays 
Table 1 Baseline characteristics and follow-up iron biomarkers in women who became pregnant (at $\mathrm{ANC} 1$ or $\mathrm{ANC} 2$ ) or remained non-pregnant (at end assessment)

\begin{tabular}{|c|c|c|c|}
\hline \multirow{2}{*}{\multicolumn{2}{|c|}{ Variable $^{1}$}} & \multirow{2}{*}{$\begin{array}{l}\text { Baseline } \\
\text { Became pregnant } \\
n=303\end{array}$} & \multirow{2}{*}{$\begin{array}{l}\text { Baseline } \\
\text { Remained non-pregnant } \\
n=712\end{array}$} \\
\hline & & & \\
\hline \multicolumn{4}{|c|}{ Demographics and medical history } \\
\hline \multicolumn{2}{|c|}{ Mean age at visit, years } & $17.0(16.0-18.0)$ & $16.0(15.0-17.0)$ \\
\hline \multicolumn{2}{|c|}{ Menarcheal at baseline (\%) } & 282/303 (93.1) & $544 / 712(76.4)$ \\
\hline \multicolumn{2}{|c|}{ Mean reproductive age (if menarcheal), years } & $3.0(2.0-4.0)[1 \mathrm{missing}]$ & $3.0(2.0-4.0)$ \\
\hline \multirow[t]{3}{*}{ Education $(\%)$} & No schooling & 199/302 (65.9) & $377 / 709(53.2)$ \\
\hline & Primary & $55 / 303(18.2)$ & $166 / 712(23.3)$ \\
\hline & Lower/higher secondary & 48/302 (15.9) & $166 / 709(23.4)$ \\
\hline \multicolumn{2}{|c|}{ Drinks alcohol (\%) } & $166 / 303(54.8)$ & $258 / 712(36.2)$ \\
\hline \multicolumn{2}{|c|}{ Sexually active $(\%)$} & $113 / 303(37.3)$ & 97/711 (13.6) \\
\hline \multicolumn{4}{|c|}{ Anthropometry } \\
\hline \multicolumn{2}{|c|}{ Mean MUAC, cm } & $24.0(22.7-25.2)$ & $23.0(21.7-24.5)$ \\
\hline \multicolumn{2}{|l|}{ MUAC $<21 \mathrm{~cm}$} & $16 / 303(5.3)$ & $107 / 712(15.0)$ \\
\hline \multicolumn{2}{|c|}{ Mean BMI, kg/m² } & $20.0(18.8-21.0)$ & $19.1(17.8-20.4)$ \\
\hline \multicolumn{2}{|c|}{$\mathrm{BMI}<18.5 \mathrm{~kg} / \mathrm{m}^{2}$} & $61 / 303(20.1)$ & $262 / 712(36.8)$ \\
\hline \multicolumn{2}{|c|}{ Anaemia and iron biomarkers } & $\mathrm{ANC1}^{2}$ & End assessment ${ }^{3}$ \\
\hline \multicolumn{2}{|c|}{$\begin{array}{l}\text { Pregnant } \mathrm{Hb}<11 \mathrm{~g} / \mathrm{dl} \text {; non-pregnant } \mathrm{Hb}< \\
12 \mathrm{~g} / \mathrm{dl}\end{array}$} & 209/303 (69.0) & $307 / 709(43.3)$ \\
\hline \multicolumn{2}{|c|}{ Mean ferritin, $\mu \mathrm{g} / \mathrm{l}$} & $\begin{array}{l}97.0(50.2-175.8)[1 \\
\text { missing }]\end{array}$ & $\begin{array}{l}52.0(28.0-91.5)[4 \\
\text { missing] }\end{array}$ \\
\hline \multicolumn{2}{|c|}{ Mean adjusted ferritin, $\mu \mathrm{g} / \mathrm{l}^{4}$} & $46.9(26.4-88.1)[1$ missing $]$ & $\begin{array}{l}37.2(18.8-65.1)[4 \\
\text { missing] }\end{array}$ \\
\hline \multicolumn{2}{|c|}{ Mean sTfR, $\mu \mathrm{g} / \mathrm{ml}$} & $3.0(2.3-4.1)[2 \mathrm{missing}]$ & $3.7(2.8-5.2)$ [6 missing] \\
\hline \multicolumn{2}{|c|}{$\begin{array}{l}\text { Ratio } \operatorname{sTfR}(\mu \mathrm{g} / \mathrm{ml}) / \log _{10} \text { ferritin }(\mu \mathrm{g} / \mathrm{l})>5.6 \text {, } \\
(\%)^{5}\end{array}$} & $34 / 301(11.3)$ & $143 / 706(20.3)$ \\
\hline \multicolumn{2}{|c|}{ Mean ZPP, $\mu \mathrm{mol} / \mathrm{mol}$ heme } & $102(87-120)$ [24 missing] & 93 (78-115) [2 missing] \\
\hline \multicolumn{2}{|c|}{ Mean body iron stores, $\mathrm{mg} / \mathrm{kg}^{4}$} & 5.9 (3.8-8.6) [2 missing] & $5.1(2.3-7.4)[6$ missing $]$ \\
\hline \multicolumn{2}{|c|}{ Mean hepcidin, $\mu \mathrm{mol} / \mathrm{ml}$} & 3.3 (1.1-7.8) [4 missing] & $3.2(1.3-6.2$ [6 missing] \\
\hline \multicolumn{2}{|c|}{ Mean CRP, mg/l } & $4.4(1.2-13.0)$ [3 missing] & $0.6(0.2-1.5)$ [5 missing] \\
\hline
\end{tabular}

MUAC mid-upper arm circumference, BMI body mass index, $s T f R$ serum transferrin receptor, CRP Creactive protein, $A N C$ antenatal clinic visit, $H b$ hemoglobin

${ }^{1}$ Brackets: interquartile range, or $\%$

${ }^{2}$ Includes pregnancies identified at, or shortly after, end assessment

${ }^{3}$ Menarcheal $n=621$; non-menarcheal $n=91$

${ }^{4}$ Ferritin estimated using an internal regression correction approach allowing for inflammation based on CRP as described by Namaste et al. [23]

${ }^{5}$ Ratio sTfR/ $\log _{10}$ ferritin provides the estimate of proportion iron deficient [21] status, as a covariate. Additional models considered interaction terms between visit and the independent variable: as none of these approached statistical significance $(P>0.05)$ they are not considered further. Statistical analyses were conducted in the R statistical environment version 3.3.1. [25].

\section{Results}

Lf samples were available for 712 non-pregnant women at end assessment (after up to 18 months of weekly iron supplementation) and for 303 women seen at ANC1 or ANC2 who had become pregnant after randomization. Lf assays were conducted for 271 at ANC1 and 241 at ANC2, providing a total of 1224 samples for Lf assay (Fig. 1). At ANC1 median and interquartile range (IQR) gestational age was 18 (14-22) weeks and at ANC2, 34 (33-35) weeks. Baseline characteristics of the non-pregnant and pregnant women are shown in Table 1 for those with an Lf measurement. Women who became pregnant were primigravidae, older and more sexually active at baseline than women who remained non-pregnant. At ANC1 prevalence 
of iron deficiency was $11.3 \%$ in pregnant women, and at end assessment $20.3 \%$ in non-pregnant women.

Mean Lf concentration for pregnant women was 806 $(65-4386) \mu \mathrm{g} / \mathrm{ml} \quad(n=271)$ and for non-pregnant 201 (66-936) $\mu \mathrm{g} / \mathrm{ml}(n=712)$. Lf distributions are shown in Supplementary file, Fig. S1. Lf concentration did not differ by menarcheal status; therefore both menarcheal groups were pooled in further analyses of the non-pregnant cohort (Supplementary file, Fig. S2). Pregnant women had higher Lf levels (difference in $\log _{10} \mathrm{Lf}=0.45,95 \%$ CI, 0.33-0.57, $P<0.0001)$ than non-pregnant women. Concentrations did not differ between ANC1 and ANC2 (Supplementary file, Fig. S2). Mean Lf concentration in pregnancy was similar to that of sexually active non-pregnant women and was independent of gestation (Fig. 2).

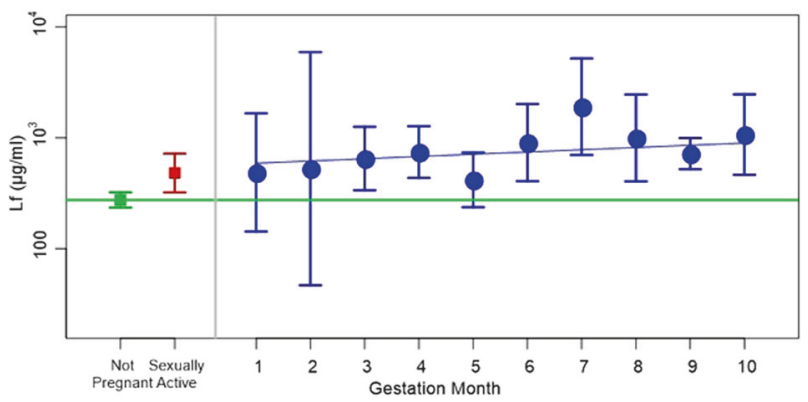

Fig. 2 Lf concentration $(\mu \mathrm{g} / \mathrm{ml})$ by gestation and in non-pregnant and sexually active women. Gestation determined by ultrasound at ANC1 in the pregnant cohort. Mean with 95\% CI for each lunar month of gestation plus values for the non-pregnant group (Non-pregnant) and the subset of non-pregnant women who were known to be sexually active at the baseline assessment. The horizontal green line indicates non-pregnant mean; blue line indicates linear regression line of log (Lf) v gestation with a random effect to allow for correlations within participants. The slope is non-significant, $P=0.14$

Fig. 3 Longitudinal Lf concentration $(\mu \mathrm{g} / \mathrm{ml})$ in 51 women seen while non-pregnant (at end assessment) and while pregnant (at ANC1 and/or ANC2). Lines join the measurements on the same woman at the three time points. Panels and colors indicate the non-pregnant Lf quartile: low, intermediate and high levels at the first assessment time. Closed circle: $\mathrm{BV}$ or $T$. vaginalis infection at the time of visit; open circle: no infection. Data are offset slightly for clarity. Shaded boxplots are included, showing the full cohort range (median, IQR, min, max)

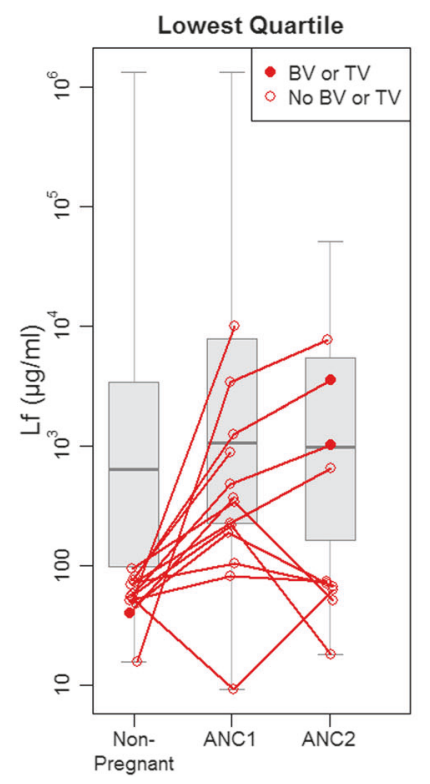

There was large variation in $\log _{10} \mathrm{Lf}$ values between individuals ranging over six orders of magnitude. The within patient/visit estimated assay SD in $\log _{10}$ Lf was 0.47 and the interclass correlation coefficient for replicate measurements was 0.90 , indicating $90 \%$ of variation was due to true differences between women or visits. The between-visit (within woman) variation SD was 0.65 , and the betweenwomen SD was 0.75 , giving a total SD between independent assessments of 1.00. The within-patient ICC was 0.57 , indicating that $\mathrm{Lf}$ values were strongly correlated over time, with individuals having levels that persisted. This is illustrated in Fig. 3, which shows the longitudinal trends in Lf concentration for the 51 women assessed at end assessment and who were identified by urine screening at that time as in very early pregnancy and then who later provided additional samples as part of the pregnant cohort at ANC1 and/or ANC2.

Some $37 \%$ of non-pregnant women had a baseline BMI $<18.5 \mathrm{~kg} / \mathrm{m}^{2}$ (Table 1). Across both pregnant and nonpregnant women, Lf concentrations increased with MUAC $(P=0.047)$ and BMI $(P=0.018)$, (Table 2$)$. The effect of sexual activity on Lf concentration in non-pregnant women remained after adjusting for $\operatorname{MUAC}(P=0.005)$ (Table 2). There was no Lf association with calendar age or reproductive age.

Prevalence of BV was $11 \%$ and $T$. vaginalis $4 \%$ at the non-pregnant end assessment, and $7 \%$ and $13 \%$, respectively, at ANC1. Lf concentration by infection category is shown in Supplementary Fig. S3. Lf levels were 6.6-fold higher in all women with BV (difference in $\log _{10} \mathrm{Lf}=0.82$, $0.63-1.00, P<0.001)$, 11.5-fold higher with $T$. vaginalis (difference in $\log _{10} \mathrm{Lf}=1.06,0.85-1.26, P<0.001$ ), and 1.8 -fold higher with vaginal discharge (difference in $\log _{10}$
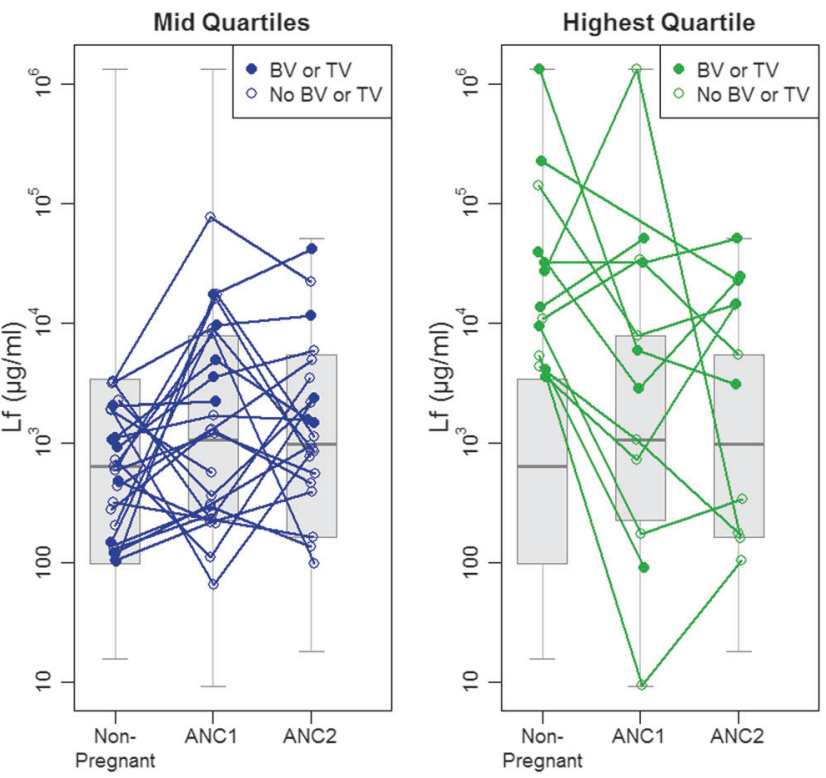
Table 2 Effect estimates of demographic, anthropometric, infection, and iron biomarkers with vaginal $\mathrm{Lf}$ concentration

\begin{tabular}{|c|c|c|c|c|c|c|c|}
\hline \multirow[b]{2}{*}{ Parameter } & \multirow[b]{2}{*}{$N^{3}$} & \multicolumn{3}{|c|}{ Adjusted for visit ${ }^{1}$} & \multicolumn{3}{|c|}{ Adjusted for visit and MUAC ${ }^{2}$} \\
\hline & & $P_{\text {visit }}{ }^{4}$ & Effect $(95 \% \mathrm{CI})$ & $P^{5}$ & $P_{\text {visit }}^{4}$ & Effect $(95 \% \mathrm{CI})$ & $P^{5}$ \\
\hline Reproductive age (per year) & 1188 & $<0.001$ & $0.01(-0.02-0.05)$ & 0.40 & $<0.001$ & $0.01(-0.03-0.04)$ & 0.67 \\
\hline Sexually active ${ }^{6}$ (yes) & 711 & NA & $0.28(0.08-0.48)$ & 0.005 & NA & $0.24(0.04-0.44)$ & 0.019 \\
\hline Alcohol (yes) & 1212 & $<0.001$ & $-0.02(-0.14-0.09)$ & 0.71 & $<0.001$ & $-0.01(-0.13-0.10)$ & 0.81 \\
\hline BMI (per $\mathrm{kg} / \mathrm{m}^{2}$ ) & 1224 & $<0.001$ & $0.03(0.01-0.06)$ & 0.018 & NA & NA & NA \\
\hline MUAC (per cm) & 1224 & $<0.001$ & $0.03(0.00-0.06)$ & 0.047 & NA & NA & NA \\
\hline No schooling (yes) & 1219 & $<0.001$ & $-0.01(-0.13-0.11)$ & 0.83 & $<0.001$ & $-0.01(-0.12-0.0)$ & 0.93 \\
\hline Nugent scores: $0-3$ & 1146 & $<0.001$ & Reference & $<0.001$ & $<0.001$ & Reference & $<0.001$ \\
\hline $4-6$ & NA & NA & $0.41(0.23-0.59)$ & NA & $<0.001$ & $0.42(0.24-0.60)$ & NA \\
\hline $7-10$ & NA & NA & $0.82(0.63-1.00)$ & NA & $<0.001$ & $0.81(0.62-1.00)$ & NA \\
\hline T. vaginalis (yes) & 1215 & $<0.001$ & $1.06(0.85-1.26)$ & $<0.001$ & $<0.001$ & $1.06(0.86-1.26)$ & $<0.001$ \\
\hline Vaginal discharge (yes) & 1221 & $<0.001$ & $0.26(0.07-0.45)$ & 0.006 & $<0.001$ & $0.26(0.08-0.45)$ & 0.006 \\
\hline Antibiotic use (yes) & 1224 & $<0.001$ & $0.12(-0.01-0.24)$ & 0.066 & $<0.001$ & $0.11(-0.01-0.24)$ & 0.075 \\
\hline Antifungal use (yes) & 1224 & $<0.001$ & $0.49(0.25-0.73)$ & $<0.001$ & $<0.001$ & $0.48(0.24-0.72)$ & $<0.001$ \\
\hline Adjusted ferritin, $\mu \mathrm{g} / \mathrm{l}^{7}$ & 1216 & $<0.001$ & $0.07(0.01-0.13)$ & 0.021 & $<0.001$ & $0.07(0.01-0.13)$ & 0.018 \\
\hline $\mathrm{sTfR}, \mu \mathrm{g} / \mathrm{ml}$ & 1216 & $<0.001$ & $-0.01(-0.15-0.13)$ & 0.86 & $<0.001$ & $0.00(-0.14-0.14)$ & 0.99 \\
\hline $\mathrm{ZPP}, \mu \mathrm{mol} / \mathrm{mol}$ heme & 1216 & $<0.001$ & $-0.03(-0.18-0.12)$ & 0.73 & $<0.001$ & $-0.02(-0.17-0.13)$ & 0.82 \\
\hline Ratio sTfR $(\mu \mathrm{g} / \mathrm{ml}) / \log _{10}$ ferritin $(\mu \mathrm{g} / \mathrm{l})$ & 1213 & $<0.001$ & $-0.08(-0.18-0.02)$ & 0.14 & $<0.001$ & $-0.07(-0.18-0.03)$ & 0.16 \\
\hline Hepcidin, $\mu \mathrm{mol} / \mathrm{ml}$ & 1211 & $<0.001$ & $0.05\left(\begin{array}{llll}0.00 & 0.09)\end{array}\right.$ & 0.040 & $<0.001$ & $0.04(0.00-0.09)$ & 0.047 \\
\hline $\mathrm{CRP}, \mathrm{mg} / \mathrm{l}$ & 1212 & $<0.001$ & $0.03(0.00-0.07)$ & 0.062 & $<0.001$ & $0.04(0.00-0.07)$ & 0.048 \\
\hline $\mathrm{Hb}, \mathrm{g} / \mathrm{dl}$ & 1219 & $<0.001$ & $-0.01(-0.45-0.43)$ & 0.96 & $<0.001$ & $-0.06(-0.49-0.38)$ & 0.81 \\
\hline Body iron stores, $\mathrm{mg} / \mathrm{kg}^{7}$ & 1213 & $<0.001$ & $0.014(0.001-0.028)$ & 0.041 & $<0.001$ & $0.014(0.000-0.028)$ & 0.042 \\
\hline
\end{tabular}

$B M I$ body mass index, $M U A C$ mid-upper-arm-circumference, $s T f R$ serum transferrin receptor,

$Z P P$ whole blood zinc protoporphyrin, $C R P$ C-reactive protein, $N A$ not applicable

${ }^{1}$ Combined data for Lf measurements at follow-up in non-pregnant (end assessment) or pregnant women (ANC1 and ANC2). Effect sizes are differences in $\log _{10} \mathrm{Lf}$ per unit, or between groups, adjusting for visit, or visit and MUAC, as fixed factors, and participant as a random effect in a linear mixed model that includes all women and visits in a single analysis

${ }^{2}$ Additional model adjusted also for MUAC assessed at ANC1 or end assessment

${ }^{3}$ Number of Lf measurements included in the analysis across all visits after missing data excluded

${ }^{4} P_{\text {visit }}: P$-value associated with the visit effect

${ }^{5}$ Significance of the effect of the variable itself-average across all visits

${ }^{6}$ Assessed at baseline and related to Lf concentration measured for non-pregnant women at end assessment only

${ }^{7}$ Ferritin estimated using an internal regression correction approach allowing for inflammation based on CRP as described by Namaste et al. [23]
Lf $=0.26,0.07-0.45, P=0.006)$ (Table 2). Receiving an antifungal prescription, predominantly for a vaginal infection, during the 6 months prior to assessment, was associated with higher Lf values $(<0.001)$. Antibiotic prescriptions, mainly for respiratory, gastrointestinal and localized infections, showed a borderline significant association $(P=0.066)$. Infection associations with $\mathrm{Lf}$ did not differ significantly after adjusting for MUAC.

Three microbiota vaginal CSTs were matched: CST I (40.4\%), III (25.8\%) and IV (33.8\%), dominated, respectively, by Lactobacillus $(L)$ crispatus, $L$. iners and a mixed community with reduced lactobacilli. The 15 bacterial genera associated with each CST category are shown in Fig. 4. Log Lf concentration differences for CST III and IV compared to CST 1 were $0.59,0.46-0.73$, and 0.58 , $0.46-0.71$, respectively $(P<0.001)$ (Supplementary file, Fig. S4).
There were significant positive trends towards increasing Lf concentration, adjusted for visit and MUAC, with serum ferritin $(P=0.018)$, serum hepcidin $(P=0.047)$ and total BIS $(P=0.042)$, with a borderline association with serum CRP concentration $(P=0.048)$ (Table 2$)$. ZPP and sTfR adjusted for MUAC were not significantly associated with Lf concentration.

\section{Discussion}

Both nutritional and iron biomarker correlates with $\mathrm{Lf}$ concentration were identified in this analysis. The higher Lf in pregnancy probably relates to exposure to sexually transmitted infections such as $T$. vaginalis consequent to regular sexual activity, as well as to higher estrogen levels [26]. No standardized methodology is available for 


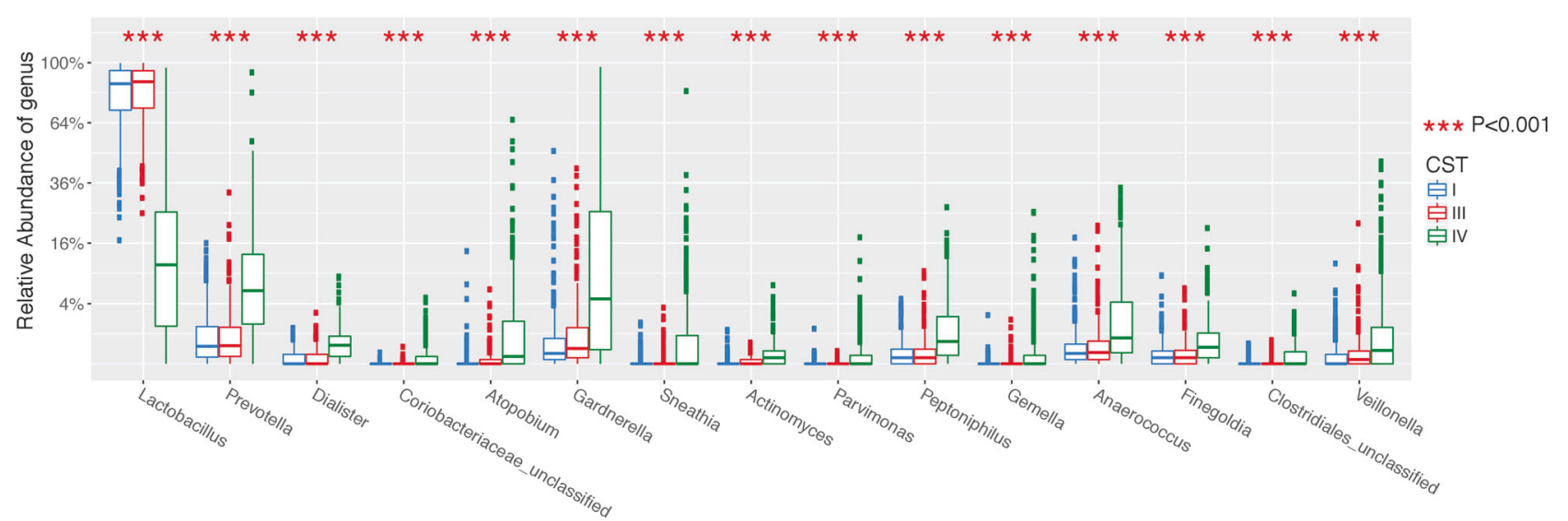

Fig. 4 Taxonomic abundance plots of bacteria associated with CST I, III and IV. Boxplots show the 15 genera most associated with CST differences and are plotted in order of significance. The centre line denotes the median, the boxes cover the 25 th and 75 th percentiles, and the whiskers extend to the most extreme data point, which is no more

measuring $\mathrm{Lf}$ in vaginal fluid and no pre-determined cutoffs are available to denote high and low values [27, 28]. For specimen collection, self-taken swabs were acceptable to the participants. Although swabs/tubes were weighed to allow adjustment for the volume of vaginal fluid sampled, this did not account for all variation between mean weights, which could be affected by hydration and seasonal effects. Normalization therefore used mean rather than individual sample weights. Despite this the assay was sufficiently sensitive to detect differences associated with participant and clinical characteristics. Lf concentrations showed a wide range in values but longitudinal profiling and analysis of the components of variation indicated women with persistently high or low Lf concentrations, maintained from before, and through, pregnancy. They may represent hyperor hypo-responsive cohorts since $\mathrm{BV}$ and $T$. vaginalis infections were identified in both categories (Fig. 3). Infection was the main factor affecting Lf concentration, but it remains unclear whether these higher Lf concentrations are helpful or detrimental. Lf is known to be a potent inhibitor of Candida albicans [29] and synergistically enhances the effects of the azole class of antifungal agents [30]. We report higher Lf concentrations in CSTs III/IV, conditions in which healthy lactobacilli are generally reduced but $L$. Iners survives. Unlike other lactobacilli, $L$. iners requires iron for growth. It probably utilizes iron released from erythrocyte destruction by Gardnerella vaginalis and flourishes during menses [31]. Its role in maintenance of iron homeostasis during infection warrants further research.

Adjustments were made for nutritional status using MUAC, which unlike BMI does not change with gestation in normal pregnancies, and mean MUAC was comparable for non-pregnant and pregnant women. Nutritional status is than 1.5 times the length of the box away from the box. Points outside the whiskers represent outlier samples. Statistical comparisons based on the Kruskal-Wallis test and all $P$-values are adjusted for multiple comparisons using the false discovery rate algorithm, where *** denotes FDR $P<0.001$

important for mucosal immunity, which may be affected by states of under- or over-nutrition. Serum Lf concentration positively correlates with insulin resistance in obese women [32]. Leptin, an adipocytokine produced by epithelial cells and adipose tissue, has a role in mucosal defenses possibly mediated by expression of IL23 [33]. The significant positive association of BMI and MUAC with vaginal Lf is consistent with leptin's influence on constitutive Lf production in young women who are still maturing.

We have shown a positive association between serum hepcidin and vaginal Lf concentration. These results may indicate a simple association of inflammation with these markers, but increased hepcidin would still impact on iron homeostasis. Hepcidin is produced by hepatocytes, but may also be locally synthesized by myeloid cells in response to pathogens, and has been observed in neutrophils migrating to tissue sites of infection [34]. There are few prior reports on hepcidin response following local, as opposed to systemic infection other than in the gastro-intestinal tract during colitis [35], and in viral keratitis [36]. The mechanisms are unclear but in vivo studies implicate host induction of local acute-phase response proteins and evidence of a hostimposed metal ion limited environment [37]. Hepcidin expression is inhibited in states of iron deficiency. Host nutritional and iron status may influence the homeostatic mechanisms controlling vaginal Lf expression. Vaginal Lf concentrations were increased in better-nourished women, yet we have previously reported that, at baseline, women who were iron deficient were more likely to have normal vaginal flora [16]. In conclusion, Lf concentrations increased with genital infection, higher BMI, MUAC, BIS and hepcidin, suggesting nutritional and iron status influence homeostatic mechanisms controlling vaginal Lf responses. Further research is needed to elucidate whether 
genital infections induce a hepcidin response, which could influence iron availability to vaginal bacteria and fungi. Figure S5 in Supplementary file 2 outlines potential pathways that could be explored.

\section{Supplementary material}

Supplementary file 1 (word doc.) includes the intention to treat comparison of Lf by trial arm. Four supplementary figures provide data on 1) Fig. S1. Lf distribution curves; 2) Fig. S2. Non-significant Lf differences for menarche/ANC visit 3); Fig. S3. Boxplots of $\mathrm{Lf}$ for six infection indicators; 4) Fig. S4. Lf in three CST by study visit.

Supplementary file 2 provides a diagram and brief description of potential systemic or local hepcidin effects with genital infection. This schema is not deduced from study results but relates to discussion points speculating on the role of hepcidin at this mucosal site.

Acknowledgements We thank the local communities, study teams, and health staff in Nanoro Health District for their co-operation; Professor Stephen Cummings, Greg Harper, and Marc Tahita for laboratory support; and Professor Brian Faragher for providing the trial data set.

Author contributions BJB was the Principal Investigator for the main RCT on iron supplementation and wrote this paper; SAR was coauthor and study statistician; LB conceived the study and was joint coauthor; SG was the clinical and field co-ordinator for the research; Lf assays were conducted in Burkina Faso by SD; hepcidin by DWS and AJG-M in the Netherlands; and microbiome by AN and CS at Northumbria University, UK. AK and SO were responsible for data management and safety monitoring; UDA and HT were members of the Scientific Advisory Committee and reviewed the paper. All authors agree to be accountable for their work.

\section{Compliance with ethical standards}

Conflicts of interest This work was supported by the National Institutes of Health (Grant Number U01HD061234-01A1; Supplementary $-05 \mathrm{~S} 1$ and -02S2), the National Institute of Child Health and Human Development / Gates Foundation, and the National Institutes of Health Office of Dietary Supplements. The grant covered salary and study costs in Burkina Faso, including salary costs for the field co-ordinator. Statistical analysis in the UK was part-funded. The contents of this publication are solely the responsibility of the authors and do not necessarily represent the views of the funding organization, which played no role in data analysis. Dorine Swinkels is Medical Director of the "Hepcidinanalysis.com" initiative (www.hepcidinanalysis.com), which aims to serve the scientific, medical, and pharmaceutical communities with high-quality hepcidin measurements. The other authors declared that they have no conflicts of interest.

Publisher's note: Springer Nature remains neutral with regard to jurisdictional claims in published maps and institutional affiliations.

Open Access This article is licensed under a Creative Commons Attribution 4.0 International License, which permits use, sharing, adaptation, distribution and reproduction in any medium or format, as long as you give appropriate credit to the original author(s) and the source, provide a link to the Creative Commons license, and indicate if changes were made. The images or other third party material in this article are included in the article's Creative Commons license, unless indicated otherwise in a credit line to the material. If material is not included in the article's Creative Commons license and your intended use is not permitted by statutory regulation or exceeds the permitted use, you will need to obtain permission directly from the copyright holder. To view a copy of this license, visit http://creativecommons. org/licenses/by/4.0/.

\section{References}

1. Baker EN, Baker HM, Kidd RD. Lactoferrin and transferrin: functional variations on a common structural framework. Biochem Cell Biol. 2002;80:27-34.

2. Jenssen H, Hancock REW. Antimicrobial properties of lactoferrin. Biochimie. 2009;91:19-29.

3. Valenti P, Rosa L, Capobianco D, Lepanto MS, Schiavi E, Cutone A, et al. Role of lactobacilli and lactoferrin in the mucosal cervicovaginal defense. Front Immunol. 2018;9:376. https://doi.org/ 10.3389/fimmu.2018.00376.

4. Delgado-Rizo V, Martínez-Guzmán MA, Iñiguez-Gutierrez L, García-Orozco A, Alvarado-Navarro A, Fafutis-Morris M. Neutrophil extracellular traps and its implications in inflammation: an overview. Front Immunol. 2017;8:81. https://doi.org/10.3389/ fimmu.2017.00081.

5. Brabin L, Brabin BJ, Gies S. Influence of iron status on risk of maternal or neonatal infection and on neonatal mortality with an emphasis on developing countries. Nutr Rev. 2013;71:528-40.

6. Ganz T. Iron and infection. Int J Hematol. 2018;107:7-15.

7. Tang L-J, De Seta F, Odreman F, Venge P, Piva C, Guaschino S, et al. Proteomic analysis of human cervical-vaginal fluids. J Proteome Res. 2007;6:2874-83.

8. Spear TS, Kendrick SR, Chen HY, Thomas TT, Bahk M, Balderas $\mathrm{R}$, et al. Multiplex immunoassay of lower genital tract mucosal fluid from women attending an urban STD clinic shows broadly increased IL1 $\beta$ and lactoferrin. PLoS One. 2011;6:e19560.

9. Novak RM, Donoval BA, Graham PJ, Boksa LA, Spear G, Hershow RC, et al. Cervicovaginal levels of lactoferrin, secretory leukocyte protease inhibitor, and RANTES and the effects of coexisting vaginoses in human immunodeficiency virus (HIV)-seronegative women with a high risk of heterosexual acquisition of HIV infection. Clin Vaccin Immunol. 2007;14:1102-7.

10. Jarosik GP, Land CB. Identification of a human lactoferrinbinding protein in Gardnerella vaginalis. Infect Immun. 2000;68:3443-7.

11. Cutone A, Rosa L, Lepanto MS, Scotti MJ, Berlutti F, Bonaccorsi di Patti MC, et al. Lactoferrin efficiently counteracts the inflammation-induced changes of the iron homeostasis system in macrophages. Front Immunol. 2017;8:705. https://doi.org/10. 3389/fimmu.2017.00705.

12. Teng CT. Lactoferrin: the path from protein to gene. Biometals. 2010;23:359-64.

13. Cohen MS, Britigan BE, French M, Bean K. Preliminary observations on lactoferrin secretion in human vaginal mucus, variation during the menstrual cycle, evidence of hormonal regulation and implications for infection with Neisseria gonorrhoeae. Am J Obstet Gynecol. 1987;157:1122-5.

14. Rebelo I, Carvalho-Guerra F, Pereira-Leite L, Quintanilha A. Lactoferrin as a sensitive blood marker of neutrophil activation in normal pregnancies. Eur J Obstet Gynaecol Reprod Biol. 1995;62:189-94.

15. Gies S, Diallo S, Roberts SA, Kazienga A, Powney M, Brabin L, et al. Effects of weekly iron and folic acid supplements on malaria risk in nulliparous women in Burkina Faso: A periconceptional, 
double-blind, randomized controlled non-inferiority trial. J Infect Dis. 2018;218:1099-109.

16. Brabin L, Roberts SA, Gies S, Nelson A, Diallo S, Stewart CJ, et al. Effects of long-term weekly iron and folic acid supplementation on lower genital tract infection - a double blind, randomised controlled trial in Burkina Faso. BMC Med. 2017;15:206. https://doi.org/10.1186/s12916-017-0967.

17. Brabin BJ, Gies S, Owens S, Claeys Y, D'Alessandro U, Tinto H, et al. Perspectives on the design and methodology of periconceptional nutrient supplementation trials. Trials. 2016;17:58.

18. Institut National de la Statistique et de la Démographie (INSD) et ICF International. Enquète Demographique et de Santé et Indicateurs Multiples du Burkina Faso 2010. Calverton, Maryland, USA: INSD et ICF International; 2012.

19. World Health Organization. Guideline: Intermittent iron and folic acid supplementation in menstruating women. Geneva: WHO; 2011.

20. Kozich JJ, Westcott SL, Baxter NT, Highlander SK, Schloss PD. Development of a dual-index sequencing strategy and cultivation pipeline for analyzing amplicon sequence data in the MiSeq Illumina sequencing platform. Appl Environ Microbiol. 2013;79:5112-20.

21. Diallo S, Roberts SA, Gies S, Rouamba T, Swinkels DW, GeurtsMoespot AJ, et al. Malaria early in the first pregnancy: potential impact of iron status. Clin Nutr. 2019;:S0261-5614:30034-2. https://doi.org/10.1016/j.clnu.2019.01.016.

22. Cook J, Flowers CH, Skikne BS. The quantitative assessment of body iron. Blood. 2003;101:3359-364.

23. Namaste SM, Rohner F, Huang J, Bhushan NL, Flores-Ayala R, Kupka R, et al. Adjusting ferritin concentrations for inflammation: biomarkers reflecting inflammation and nutritional determinants of anemia (BRINDA) project. Am J Clin Nutr. 2017;106:359S-371S.

24. Kroot JCC, Laarakkers CM, Geurts-Moespot A, Grebenchtchikov $\mathrm{N}$, Pickkers $\mathrm{P}$, van Ede A, et al. Immunochemical and mass spectrometry-based serum hepcidin assays for a variety of iron metabolism disorders. Clin Chem. 2010;56:1570-9.

25. R Foundation for Statistical Computing. R: a language and environment for statistical computing. R Core Team: Vienna, Austria; 2016.

26. Teng CT. Factors regulating lactoferrin gene expression. Biochem Cell Biol. 2006;84:263-7.

27. Rein MF, Shih LM, Miller JR, Guerrant RL. Use of a lactoferrin assay in the differential diagnosis of female genital tract infections and implications for the pathophysiology of bacterial vaginosis. Sex Transm Dis. 1996;23:517-21.

28. Goldenberg RL, Andrews WW, Guerrant RL, Newman M, Mercer B, Iams J, et al. The Preterm Prediction Study: cervical lactoferrin concentration, other markers of lower genital tract infection, and preterm birth. Am J Obstet Gynecol. 2000;182:631-5.

29. Bairwa G, Jung WH, Kronstad JW. Iron acquisition in fungal pathogens of humans. Metallomics. 2017;9:215-27.

30. Kobayashi T, Kakeya H, Miyazaki T, Izumikawa TK, Yanagihara $\mathrm{K}$, Ohno H, et al. Synergistic antifungal effect of lactoferrin with azole antifungals against Candida albicans and a proposal for a new treatment method for invasive candidiasis. Jpn J Infect Dis. 2011;64:292-6.

31. Santos Santiago GL, Tency I, Verstraelen H, Verhelst R, Trog M, Temmerman M, et al. Longitudinal qPCR study of the dynamics of L.crispatus, L.iners, A, vaginae (sialidase positive), G.vaginalis, and P. bivia in the vagina. PLoS One. 2012;7:e45281 https:// doi.org/10.1371/journal.pone.0045281e45281. (2012).

32. Mayeur S, Veilleux A, Pouliot Y, Lamarche B, Beaulieu F, Hould FS. et al. Plasma lactoferrin levels positively correlate with insulin resistance despite an inverse association with total adiposity in lean and severely obese patients. PLoS One. 2016;11:eo166138 https://doi.org/10.1371/journal.pone.0166138.

33. Madan R, Guo X, Naylor C, Buonomo EL, Mackay D, Noor Z, et al. Role of leptin-mediated colonic inflammation in defense against Clostridium difficile colitis. Infect Immun. 2014;82:341-9.

34. Peyssonnaux C, Zinkernagel AS, Datta V, Lauth X, Johnson RS, Nizet V. TLR4- dependent hepcidin expression by myeloid cells in response to bacterial pathogens. Blood. 2006;107:3727-32.

35. Verma S, Cherayil BJ. Iron and inflammation-the gut reaction. Metallomics. 2017;9:101-11.

36. Mohammed I, Abedin A, Tsintzas K, Abedin SA, Otri AM, Hopkinson A, et al. Increased expression of hepcidin and toll-like receptors 8 and 10 in viral keratitis. Cornea. 2011;30:899-904.

37. Morrow BJ, Liu X, Dodge IL, Vitiello A, Ward CK, Shaw KJ. Simultaneous analysis of host and pathogen interactions during an in vivo infection reveals local induction of host acute phase response proteins, a novel bacterial stress response, and evidence of a host-imposed metal ion limited environment. Cell Microbiol. 2004;6:849-65. 